\title{
Osteogenesis with Cells and Scaffold.
}

\author{
Akihiro Umezawa'), Guoping Chen²), and Kohei Tsuchiya1.
}

\author{
${ }^{1)}$ Department of Reproductive Biology and Pathology, \\ National Institute for Child Health and Development \\ ${ }^{2}$ Biomaterials Center, National Institute for Materials Science, Ibaragi, 305-0044, Japan.
}

\begin{abstract}
A novel web-like structured, biodegradable, hybrid sheet has been developed for bone tissue engineering by preparing knitted poly(DL-lactic-co-glycolic acid) (PLGA) sheets with collagen microsponges in their openings. The PLGA skeleton facilitated formation of the hybrid sheets into desired shapes, and the collagen microsponges in the pores of the PLGA sheet promoted cell adhesion and uniform cell distribution throughout the sheet. A large number of osteoblasts established from marrow stroma adhered to the scaffolds and generated the desired shaped bone in combination with this novel sheets. The web-like structured novel sheet show promise for use as a tool for custom-shaped bone regeneration in basic research on osteogenesis and for the development of therapeutic applications.
\end{abstract}

\section{Introduction}

Tissues originating in the mesoderm include blood cells, blood vessels, heart tissues, bone, cartilage, adipose tissue, skeletal muscle, tendon, and fibroblasts. Blood cells in bone marrow are the system that created the concept of stem cells, and bone marrow includes another cell group possessing various adhering properties, i.e. mesenchymal cells. In the field of mesenchymal cell research to date, there are reports of a commitment successfully imparted somewhat selectively to osteoblasts ${ }^{1)}$, chondrocytes $^{2}$, adipocytes ${ }^{3)}$, and neuron ${ }^{4,5)}$ (Fig. 1). Recently, We have reported successful derivation of myocardial cells from mesenchymal cells under in vivo conditions [5]. In vivo differentiation was also successfully induced in skeletal muscle cells, which are the same striated muscle as myocardial cells. These results in skeletal muscle suggest that even for organs with a satellite cell regenerative system, the marrow has an organ maintenance mechanism that serves as backup. Thus, there is a newfound major potential for mesenchymal stem cells as another type of stem cell deeply rooted in the marrow, and there is a striking increase in reports on this topic.

When the existence of stem cells was ascertained, the next major issue was the problem of their purification of multipotent stem cells (Fig. 2). One technique is the use of an organ specific promoter. Nerve cells, like myocardial cells, are deemed to cease proliferation when terminal differentiation is reached, but the existence of nerve stem cells has also been demonstrated recently. To isolate human nerve stem cells, a plasmid coding for green fluorescent protein (GFP) under control of Nestin and Musashi 1 enhancer/promoters expressing in nerve stem cells was introduced to cells isolated from human brain tissue. GFP-expressing cells were then collected by a fluorescence-activated cell sorter. These cells were reported to complete cell division and differentiate into mature nerve cells. The same approach can be contemplated for the heart. Molecules expressing specifically in myocardial cells include a-myosin heavy chain, long used for their heart specific molecular expression; as well as cardiac a-actin, cardiac myosin light-chain 2, cardiac troponin $\mathrm{T}$, and $\mathrm{Na} / \mathrm{Ca}$ exchanger gene 1 . In addition to cells, scaffold is essential for cell-based therapy or

Correspondence to: Akihiro Umezawa, M.D., Ph.D.Department of Reproductive Biology and Pathology, National Research Institute for Child Health and Development 3-35-31 Taishido, Setagaya, Tokyo, 154-8567 Japan,Tokyo, 160-8582, JAPAN regenerative medicine. Collagen hybridized PLGA sponge have been generated as a scaffold both for cartilage tissue engineering with mature bovine chondrocytes ${ }^{6,7)}$ and for bone tissue engineering with osteoblasts isolated from marrow cells ${ }^{8)}$. The sponges organize good cartilage and bone tissue when the cells fill the pores of the scaffold. Uniform distribution of the cells throughout the scaffolds is imperative for the development of homogeneous tissue, but special seeding techniques, such as stir flask culture or perfusion bioreactor culture, are required to reliably produce an even distribution. Since simple static seeding methods tend to produce an uneven distribution with large patches of cells on the surface, we hybridized collagen microsponges with PLGA sheets. The sheets have a collagen fiber network in the openings of the PLGA fiber sheets. The fact that the sheets trap cells, that they can be laminated or rolled to control their shape for tissue engineering, and that they also have the capacity to supply minerals by depositing apatite particulates on the surface of collagen microsponges is a great advantage. In this study, we showed that when the novel web-like structured sheets were used as a scaffold for bone tissue engineering an even cell distribution and control of its shape were achieved.

\section{Scaffold fabrication \\ Materials and Methods}

The hybrid sheet was prepared by allowing collagen microsponges to form in the openings of PLGA knitted sheets as previously described7). A knitted Vicryl sheet made of polylactin 910 (a 90:10 copolymer of glycolic acid and lactic acid), was immersed in a type I bovine collagen acidic solution ( $\mathrm{pH}$ 3.2, 0.5 wt\%, Koken Co., Ltd., Tokyo, Japan), and frozen at $-80^{\circ} \mathrm{C}$ for 12 $\mathrm{hr}$. It was then freeze-dried under a vacuum of 0.2 Torr for $24 \mathrm{hr}$ to allow the formation of collagen microsponges. The collagen microsponges were further cross-linked by treatment with glutaraldehyde vapor saturated with a $25 \%$ aqueous glutaraldehyde solution at $37^{\circ} \mathrm{C}$ for $4 \mathrm{hr}$. After the cross-linking, the sponge was treated with a $0.1 \mathrm{M}$ aqueous glycine solution to block unreacted aldehyde groups. After washing with deionized water and freezedrying, the collagen-hybridized PLGA (PLGA/COL) sheet was complete. The sheets were sterilized with ethylene oxide for cell culture. 
International symposium of Maxillofacial \& Oral Regenerative Biology in Okayama 2005

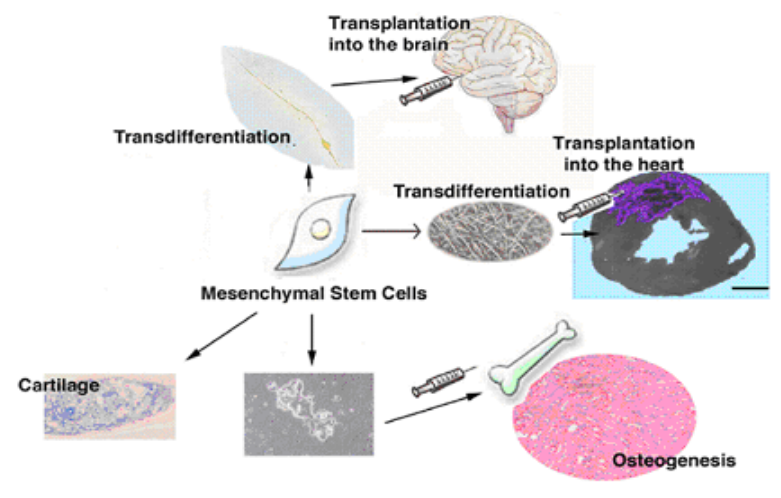

Fig. 1 Differentiation potential of mesenchymal stem cells

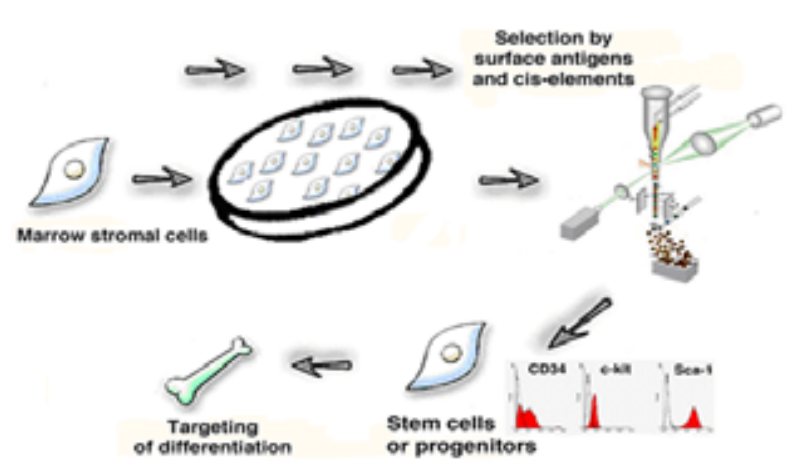

Fig. 2. Strategy for selection of stem cells or progenitors.
Cell seeding of a PLGA/COL sheet

KUSA-A1 cells were cultured in the M061101 medium (okada@med-shirotori.co.jp, MED SHIROTORI Co., Ltd., Tokyo) ${ }^{8,9}$. A sheet was placed onto a $100 \mathrm{~mm}$ culture dish (Falcon) and covered with a silicone rubber framework. A $2 \mathrm{~mL}$ volume of KUSA-A1 cell suspension having a density of 5x106/mL was dropped onto the $4 \mathrm{~cm} 2$ of PLGA/COL sheet. After cultivation for $6 \mathrm{hr}$, the sheet was flipped and re-seeded with the same number of cells on the reverse side. For comparison, a KUSA-A1 suspension (1x107 cells/ml) was injected into collagen hybrid PLGA sponges, and the injected sponges were incubated at $37^{\circ} \mathrm{C}$ for more than $30 \mathrm{~min}$. The sponges were then transplanted into the subcutaneous tissue of $\mathrm{C} 3 \mathrm{H}$ mice.

\section{Custom-shaped bone formation in mice}

With the aim of producing long bone, cylinder-like bone was formed by rolling. KUSA-A1-seeded sheets around a silicone rod $3 \mathrm{~mm}$ in diameter. The ends of the sheets were hemmed with 4-0 Vicryl dissolvable stitches. The rolled sheets were transplanted into subcutaneous tissue for 4 weeks, flat or after knotting them. Tissue-engineered phalanges were formed in a similar manner. KUSA-A1-seeded sheets were wrapped around a silicone rubber block trimmed in advance to the shape of the distal phalanx and transplanted into subcutaneous tissue. After cultivation in syngeneic C3H/He mice, NOD/SCID mice, and NOD/SCID/IL2-receptor- knock-out immunodeficient mice (NOG), the specimens were extracted and examined histologically.

\section{Results and Discussion}

Custom-shaped osteogenesis with PLGA/COL sheets

Since cylinder shaped scaffolds are more suitable for segmental long bone defects, the cell-seeded sheets were rolled up around a hollow silicone rod $3 \mathrm{~mm}$ in diameter immediately before implantation into subcutaneous tissue. After 4 weeks of in vivo cultivation, cylinder shaped bone had formed. Its outer circumference was covered with a fibrous layer, and ubiquitous vascular channels connected to it. We then attempted to produce bone of more complex shapes. The rolled sheets were knotted and transplanted into subcutaneous tissue, and uniquely shaped knots of bone were created. The cell-seeded sheets wrapped around silicone rubber blocks conformed well to the shape of the distal phalanx when removed from the mouse after 4 weeks.

Validity of the PLGA/COL hybrid sheet as a scaffold for bone tissue engineering

Biomaterials are essential for bone tissue engineering, and whether permanent or biodegradable, naturally occurring or synthetic, they must be biocompatible and ideally should be osteoinductive and osteoconductive. We employed a PLGA/COL hybrid, one of the most promising scaffolds for osteogenesis. High-molecular weight molecules, such as PLA and PGA, and inorganic materials, such as ceramics ${ }^{10}$, hydroxyapatite, and tricalcium phosphate (TCP), have been evaluated as scaffolds for osteogenesis. Hydroxyapatite and TCP efficiently conduct osteoprogenitor cells into themselves and become highly integrated with adjoining native bone, i.e., they have excellent osteoconductivity and osseointegration properties. Meanwhile, they do not induce osteogenic differentiation, i.e., they lack osteoinductivity. Moreover, there are problems associated with their slow biodegradability and their association with inflammation due to immunologic reactions.

Human MSCs have a limited life span and thus it is difficult to obtain a large number of cells. We attempted to extend the life span of human mesenchymal stem cells by transferring human telomerase reverse transcriptase and human papillomavirus E6 and E7 genes ${ }^{4,11,12)}$. The cells expressing TERT, E6, and E7 successfully proliferated over 80 population doublings. Moreover, the cells retained the pluripotency into adipocytes, myocytes, and osteocytes in vitro and in vivo. A large number of human cells with the prolonged life span established from marrow stroma adhered to the scaffolds and generated the desired shaped bone in combination with this novel sheets. The web-like structured novel sheet show promise for use as a tool for custom-shaped bone regeneration in basic research on osteogenesis and for the development of therapeutic applications.

\section{References}

1) Umezawa A, Tachibana K, Harigaya K, Kusakari S, Kato S, Watanabe YTakano T: Colony-stimulating factor 1 expression is down-regulated during the adipocyte differentiation of $\mathrm{H}$ 1/A marrow stromal cells and induced by cachectin/tumor necrosis factor. Mol Cell Biol, 11: 920-927, 1991.

2) Muraglia A, Corsi A, Riminucci M, Mastrogiacomo M, Cancedda R, Bianco P, Quarto R: Formation of a chondroosseous rudiment in micromass cultures of human bonemarrow stromal cells. J Cell Sci, 116: 2949-55, 2003.

3) Gronthos S, Chen S, Wang CY, Robey PG, Shi S: Telomerase accelerates osteogenesis of bone marrow stromal stem cells by upregulation of CBFA1, osterix, and osteocalcin. J Bone Miner Res, 18: 716-22, 2003.

4) Mori $T$, Kiyono $T$, Imabayashi $H$, Takeda $Y$, Tsuchiya $K$, Miyoshi S, Makino H, Matsumoto K, Saito H, Ogawa S, Sakamoto M, Hata J, Umezawa A: Combination of hTERT and Bmi-1, E6 or E7 induce prolongation of the life span of 
J.Hard Tissue Biology.14(2)Proceeding,2005

bone marrow stromal cells from an elderly donor without affecting their neurogenic potential. Mol Cell Biol, 25: 51835195, 2005.

5) Kohyama J, Abe H, Shimazaki T, Koizumi A, Nakashima K, Gojo S, Taga T, Okano H, Hata J, Umezawa A: Brain from bone: efficient "meta-differentiation" of marrow stromaderived mature osteoblasts to neurons with Noggin or a demethylating agent. Differentiation, 68: 235-44, 2001.

6) Sato T, Chen G, Ushida T, Ishii T, Ochiai N, Tateishi T: Tissueengineered cartilage by in vivo culturing of chondrocytes in PLGA-collagen hybrid sponge. Materials Science and Engineering C, 17: 83-89, 2001.

7) Chen G, Sato T, Ushida T, Hirochika R, Tateishi T: Redifferentiation of dedifferentiated bovine chondrocytes when cultured in vitro in a PLGA-collagen hybrid mesh. FEBS Lett, 542: 95-9, 2003.

8) Ochi K, Chen G, Ushida T, Gojo S, Segawa K, Tai H, Ueno K, Ohkawa H, Mori T, Yamaguchi A, Toyama Y, Hata J, Umezawa A: Use of isolated mature osteoblasts in abundance acts as desired-shaped bone regeneration in combination with a modified poly-DL-lactic-co-glycolic acid (PLGA)-collagen sponge. J Cell Physiol, 194: 45-53, 2003.

9) Tsuchiya K, Mori T, Chen G, Ushida T, Tateishi T, Matsuno T, Sakamoto M, Umezawa A: Custom-shaping system for bone regeneration by seeding marrow stromal cells onto a weblike biodegradable hybrid sheet. Cell Tissue Res, 316: 14153, 2004.

10) Ohgushi H, Dohi Y, Katuda T, Tamai S, Tabata S, Suwa Y: In vitro bone formation by rat marrow cell culture. J Biomed Mater Res, 32: 333-40, 1996.

11) Takeda $Y$, Mori T, Imabayashi H, Kiyono T, Gojo S, Miyoshi S, Hida N, Ita M, Segawa K, Ogawa S, Sakamoto M, Nakamura S, Umezawa A: Can the life span of human marrow stromal cells be prolonged by bmi-1, E6, E7, and/or telomerase without affecting cardiomyogenic differentiation? J Gene Med : DOI: 10.1002/jgm, 583, 2004.

12) Terai M, Uyama T, Sugiki T, Li XK, Umezawa A, Kiyono T: Immortalization of Human Fetal Cells: The Life Span of Umbilical Cord Blood-derived Cells Can Be Prolonged without Manipulating p16INK4a/RB Braking Pathway. Mol Biol Cell, 16: 1491-1499, 2005. 